\title{
The Novel CYP2A6 Inhibitor, DLCl-1, Decreases Nicotine Self-Administration in Mice
}

\author{
Yen-Chu Chen, James P. Fowler, Jing Wang, Christy J. W. Watson, Yasmine Sherafat, \\ Andres Staben, Philip Lazarus, Travis T. Denton, and (1) Christie D. Fowler \\ Department of Neurobiology and Behavior, University of California Irvine, Irvine, California (Y.-C.C., J.P.F., Y.S., A.S., C.D.F.); and \\ Department of Pharmaceutical Sciences, College of Pharmacy and Pharmaceutical Sciences, Washington State University, \\ Spokane, Washington (J.W., C.J.W.W., P.L., T.T.D.)
}

Received June 6, 2019; accepted October 16, 2019

\section{ABSTRACT}

During tobacco and e-cigarette use, nicotine is mainly metabolized in the human liver by cytochrome P450 2A6 (CYP2A6). Given that a slower CYP2A6 metabolism has been associated with less vulnerability to develop nicotine dependence, the current studies sought to validate a novel CYP2A6 inhibitor, (5-(4-ethylpyridin-3-yl)thiophen-2-yl)methanamine (DLCl-1), for its effects on intravenous nicotine self-administration. Male and female mice were trained to self-administer nicotine across daily sessions. Once stable responding was achieved, DLCl-1 or vehicle control was administered prior to nicotine sessions. We found that the lower $25 \mathrm{mg} / \mathrm{kg}$ and moderate $50 \mathrm{mg} / \mathrm{kg}$ doses of $\mathrm{DLCl}-1$ induced a significant decrease in nicotine intake for both males and females. DLCl-1 was further shown to be more effective than a moderate $1 \mathrm{mg} / \mathrm{kg}$ dose of bupropion on reducing nicotine intake and did not exert the adverse behavioral effects found with a high $75 \mathrm{mg} / \mathrm{kg}$ dose of bupropion. Although mice treated with $\mathrm{DLCl}-1$ self-administered significantly less nicotine, similar nicotine-mediated behavioral effects on locomotion were observed. Together, along with the analysis of nicotine metabolites during self-administration, these findings support the contention that blocking hepatic nicotine metabolism would allow for similar activation of nicotinic acetylcholine receptors at lower nicotine doses. Moreover, these effects of DLCl-1 were specific to nicotine self-administration, as $\mathrm{DLCl}-1$ did not result in any behavioral changes during food self-administration. Taken together, these studies validate DLCl1 as a novel compound to decrease nicotine consumption, which may thereby promote tobacco and nicotine product cessation.

\section{SIGNIFICANCE STATEMENT}

Current pharmacological approaches for nicotine and tobacco cessation have only been able to achieve limited efficaciousness in promoting long-term abstinence. In this work, we characterize the effects of a novel compound, (5-(4-ethylpyridin-3-yl)thiophen-2-yl)methanamine (DLCl-1), which inhibits the main enzyme that metabolizes nicotine, and we report a significant decrease in intravenous nicotine self-administration in male and female mice, supporting the potential of $\mathrm{DLCl}-1$ as a novel tobacco cessation pharmacotherapeutic.

\section{Introduction}

Tobacco addiction remains a leading preventable cause of disease and death worldwide, despite a high percentage of individuals expressing a desire to quit (Centers for Disease Control and Prevention (CDC), 2008). Nicotine, the main psychoactive component in tobacco, exerts its reinforcing effects by acting on neuronal nicotinic acetylcholine receptors (nAChRs) in the brain (Albuquerque et al., 1995; Léna and Changeux, 1998; Tuesta et al., 2011). Although tobacco cigarette

This work was supported by the National Institutes of Health National Institute on Drug Abuse [Grants DA032543 and DA039658] to C.D.F. National Institutes of Health National Institute of Environmental Health Sciences [Grant ES025460] to P.L.; University of California Irvine to C.D.F.; Health Sciences and Services Authority of Spokane, WA [Grant WSU002292] to Washington State University (WSU) College of Pharmacy and Pharmaceutical Sciences; and WSU College of Pharmacy and Pharmaceutical Sciences to T.T.D. and P.L.

https://doi.org/10.1124/jpet.119.260653. use has declined recently, this has unfortunately been contrasted with a dramatic increase in the use of e-cigarettes, which deliver vaporized nicotine (GBD, 2017). Although e-cigarettes have been promoted as tobacco cessation aids, product consumption by never-smokers has raised significant concerns for addiction liability, especially among youth (Etter and Bullen, 2014; Gornall, 2015; Primack et al., 2015).

In humans, the majority of nicotine is metabolized to nicotine- $\Delta^{1^{\prime}-5^{\prime}}$-iminium ion by the hepatic enzyme, cytochrome P450 2A6 (CYP2A6), followed by the conversion to cotinine by either CYP2A6 itself, or through the subsequent action of aldehyde oxidase or other enzymes (Ray et al., 2009; Raunio and Rahnasto-Rilla, 2012; von Weymarn et al., 2012). Allelic variation in the gene encoding for CYP2A6 in humans has been associated with altered nicotine metabolism, resulting in a differential vulnerability to nicotine dependence, related to the rate of metabolism (Ray et al., 2009; Thorgeirsson et al., 2010). The CYP2A6 gene is highly polymorphic with allelic

ABBREVIATIONS: 3HC, trans-3'-hydroxycotinine; CYP2A6, cytochrome P450 2A6; DLCI-1, (5-(4-ethylpyridin-3-yl)thiophen-2-yl)methanamine; FR5TO20 sec schedule, fixed-ratio 5, 20-second schedule of reinforcement; LC, liquid chromatography; MS, mass spectrometry; nAChR, nicotinic acetylcholine receptor; UPLC, ultra-pressure LC. 
variants resulting in either lower or increased metabolic function (Mwenifumbo et al., 2008; Thorgeirsson et al., 2010; Raunio and Rahnasto-Rilla, 2012). Importantly, individuals with a slow metabolizer CYP2A6 genotype are less vulnerable to develop tobacco dependence than normal metabolizers (Audrain-McGovern et al., 2007; Thorgeirsson et al., 2010; Bloom et al., 2011). It is predicted that a slower metabolism would result in more prolonged actions of nicotine following drug consumption. Therefore, with lower levels of nicotine intake, the amount of drug remaining in the system would accumulate over time, and thus, less drug would be required to achieve sustained activation of $\mathrm{nAChRs}$ in mesolimbic brain pathways mediating reinforcement (Tuesta et al., 2011; Fowler and Kenny, 2014). However, under conditions of increasing nicotine intake, activation of the pathways regulating the aversive properties of the drug, such as the medial habenulo-interpeduncular pathway, would be more readily engaged, resulting in a downward behavioral titration of drug consumption (Fowler et al., 2011; Fowler and Kenny, 2014). Supporting this contention, slow metabolizers have been found to consume less nicotine per day, experience less withdrawal symptoms, and are more successful in quitting smoking (Strasser et al., 2007; Patterson et al., 2008; Rodriguez et al., 2011; Liakoni et al., 2019). Conversely, individuals with a higher rate of metabolism exhibit greater levels of nicotine intake, withdrawal symptoms, and drug craving (Strasser et al., 2011; Chen et al., 2018a; Liakoni et al., 2019), which may thereby increase the likelihood of relapse during cessation. In a study with the cessation therapeutic bupropion, it was found that individuals with a slow metabolism are similarly successful in achieving abstinence under placebo or bupropion conditions, whereas fast metabolizers show greater benefit from bupropion treatment (Patterson et al., 2008). Given these findings in humans and the consideration that individuals with allelic variation appear to function normally despite having a reduced CYP2A6 metabolism, drug development efforts to limit nicotine metabolism represent an exciting opportunity for therapeutic modulation. Indeed, initial drug validation efforts along these lines have proven to be effective in smokers. Methoxsalen was shown to inhibit CYP2A6, and, in smokers, methoxsalen decreased nicotine metabolism and reduced cigarette consumption (Sellers et al., 2000). Conceptually, this approach could lead to a significant reduction in tobacco product use, thereby limiting exposure to harmful constituents mitigating adverse health effects, and/or provide the means for an individual to gradually reduce intake over time to eventually achieve full smoking cessation, potentially in combination with other therapeutics.

Recently, we developed a potent and selective CYP2A6 inhibitor, (5-(4-ethylpyridin-3-yl)thiophen-2-yl)methanamine (DLCI-1), which was shown to exhibit favorable metabolic stability and half-life in human liver microsomes and adheres to Lipinski's rule of five for drug likeness (Denton et al., 2018). In this study, we examined whether DLCI-1 would be effective in mediating nicotine consumption in both males and females in a mouse model. The intravenous nicotine self-administration procedure was used, as it allows for the most direct and reliable measure of nicotine reinforcement in mice (Fowler and Kenny, 2011). We also assessed the effects of the approved smoking cessation therapeutic, bupropion, on nicotine self-administration, as a means of comparing the relative effectiveness of DLCI-1 in reducing nicotine intake.
As a secondary behavioral measure, mice were examined for nicotine-mediated changes in locomotion. Finally, to ensure that DLCI-1 was selective for decreasing nicotine intake, as opposed to a general attenuation of lever-pressing behavior, the effect of DLCI-1 was assessed during food selfadministration.

\section{Materials and Methods}

Animals. Male and female wild-type C57BL/6J mice were derived from breeders in our laboratory animal facilities or were purchased from The Jackson Laboratory (Bar Harbor, ME). Mice were maintained in an environmentally controlled vivarium on a 12-hour reversed light/dark cycle. Food and water were provided ad libitum until behavioral training commenced. During food training, subjects were mildly food restricted to $85 \%$ to $90 \%$ of their free-feeding bodyweight, and water was provided ad libitum. All experiments were conducted in strict accordance with the National Institutes of Health Guide for the Care and Use of Laboratory Animals and were approved by the Institutional Animal Care and Use Committee at the University of California, Irvine.

Drugs. (-)-Nicotine hydrogen tartrate salt (0215355491; MP Biomedicals, Santa Ana, CA) was dissolved in 0.9\% sterile saline and adjusted to $\mathrm{pH}$ 7.4. The CYP2A6 inhibitor, DLCI-1, was prepared as the hydrochloride salt, as previously described (Denton et al., 2018), and then dissolved in $0.9 \%$ sterile saline, adjusted to $\mathrm{pH} 7.4$, and administered at a dose of 25 or $50 \mathrm{mg} / \mathrm{kg}$. Doses were derived by combining the generally accepted dose of $5 \mathrm{ml} / \mathrm{kg}$ and the $\mathrm{IC}_{50}$ value of the compound against CYP2A6 (Denton et al., 2018). Both DLCI-1 and vehicle were administered by mouth via oral gavage at a volume of $0.1 \mathrm{ml}$. Bupropion (B102; Sigma Aldrich, St. Louis, MO) was dissolved in $0.9 \%$ sterile saline and administered at a dose of 1 or $75 \mathrm{mg} / \mathrm{kg}$, which was injected intraperitoneally at a volume of $10 \mathrm{ml} / \mathrm{kg}$. For liquid chromatography (LC)-mass spectrometry (MS) analysis of blood samples, nicotine-methyl-D3 and cotinine-methyl-D3, trans3 '-hydroxycotinine-methyl-D3, and standards (nicotine, cotinine and trans-3'-hydroxycotinine) were obtained from Toronto Research Chemicals (North York, Canada).

Intravenous Nicotine Self-Administration. Subjects were food trained and then surgically catheterized, as previously described (Fowler et al., 2011; Chen et al., 2018b). Briefly, mice were anesthetized with an isoflurane (1\%-3\%)/oxygen vapor mixture and prepared with intravenous catheters. Catheters consisted of a 6-cm length of silastic tubing fitted to a guide cannula (Plastics One, Roanoke, VA), bent at a curved right angle, and encased in dental acrylic. The catheter tubing was passed subcutaneously from the animal's back to the right jugular vein, and a 1-cm length of the catheter tip was inserted into the vein and tied with surgical silk suture. Following the surgical procedure, animals were allowed $\geq 48$ hours to recover from surgery, and then provided access to respond for food reward until criteria were achieved. Mice were then permitted to acquire intravenous nicotine self-administration during 1-hour daily sessions, 6 to 7 days per week, at the standard training dose of nicotine $(0.03 \mathrm{mg} / \mathrm{kg} /$ infusion $)$ for 8 days, and were then transitioned onto the moderate $0.1 \mathrm{mg} / \mathrm{kg}$ per infusion dose of nicotine. This dose was chosen because mice exhibit the highest level of responding for this dose across the dose-response curve, and it also results in a similar level of intake as that found at higher doses with behavioral titration via selfadministration (Fowler and Kenny, 2011). Nicotine was delivered through tubing into the intravenous catheter by a Razel syringe pump (Med Associates, Fairfax, VT). Each session was performed using two retractable levers (one active, one inactive). Completion of the response criteria on the active lever resulted in the delivery of an intravenous nicotine infusion $[0.03 \mathrm{ml}$ infusion volume; fixed-ratio 5 , time-out 20-second schedule of reinforcement (FR5TO20 sec schedule)]. Responses on the inactive lever were recorded but had no scheduled consequences. 
After acquisition and achieving stable responding on the $0.1 \mathrm{mg} / \mathrm{kg}$ per infusion dose, subjects were examined for the effects of DLCI-1 or bupropion on nicotine intake. For Cohort 1 ( $n=5$ males), mice were orally administered DLCI-1, or vehicle control, 30 minutes prior to each session, utilizing a within-subject Latin-square design. Following these sessions, mice were placed into the open field for locomotor assessments (see below). In between each DLCI-1 or vehicle administration session, subjects were provided baseline days to ensure stable, reinstated responding prior to the next dose. For Cohort $2(n=$ 7 males, $n=6$ females), mice were treated as above, but blood was drawn immediately following each session to examine the levels of nicotine metabolites. After re-establishing baseline responding, mice were then injected with bupropion, or vehicle control, 20 minutes prior to each session utilizing a within-subject Latin-square design. In between each bupropion or vehicle administration session, subjects were provided baseline days to ensure stable, reinstated responding prior to the next dose. Catheters were flushed daily with physiologic sterile saline solution $(0.9 \% \mathrm{w} / \mathrm{v})$ containing heparin $(100 \mathrm{U} / \mathrm{ml})$. Catheter integrity was verified with the ultrashort-acting barbiturate anesthetic Brevital (2\%, methohexital sodium; Eli Lilly, Indianapolis, IN) at the end of the study. Behavioral responses were automatically recorded by MedAssociates software.

In summary, the experimental timeline was as follows: Food selfadministration $(5+$ days $) \rightarrow$ intravenous surgery and recovery $(3+$ days $) \rightarrow$ nicotine self-administration: acquisition at the $0.03 \mathrm{mg} / \mathrm{kg}$ per infusion dose ( 8 days) $\rightarrow$ self-administration of the moderate $0.1 \mathrm{mg} / \mathrm{kg}$ per infusion nicotine dose $(6$ days $) \rightarrow$ first DLCI-1 dose session (vehicle, 25 or $50 \mathrm{mg} / \mathrm{kg}$, according to Latin-square design, prior to the self-administration session), immediately followed by either a locomotor test (Cohort 1 ) or blood draw (Cohort 2) $\rightarrow$ baseline self-administration (2+ days) $\rightarrow$ second DLCI-1 dose session, immediately followed by either a locomotor test (Cohort 1) or blood draw (Cohort 2) $\rightarrow$ baseline self-administration (2+ days) $\rightarrow$ third DLCI-1 dose session, immediately followed by either a locomotor test (Cohort 1) or blood draw (Cohort 2) $\rightarrow$ Cohort 1 end. Cohort 2 mice then proceeded to the following: baseline self-administration (2+ days) $\rightarrow$ first bupropion dose session (vehicle, 1 or $75 \mathrm{mg} / \mathrm{kg}$, according to Latinsquare design, prior to the self-administration session) $\rightarrow$ baseline self-administration $(2+$ days $) \rightarrow$ second bupropion dose session $\rightarrow$ baseline self-administration $(2+$ days $) \rightarrow$ third bupropion dose session. One female developed a leaky catheter during the bupropion dosing, so she was removed, and two additional females were assessed only with bupropion to achieve sufficient subject numbers.

Ultra-Pressure Liquid Chromatography-Mass Spectrometry. Immediately following the self-administration session, blood samples were collected from the mice via the facial vein, placed into a microcentrifuge tube with $20 \mu$ l EDTA ( $500 \mathrm{mM}, \mathrm{pH} 8.0$ ) on ice, and centrifuged at $3000 \mathrm{~g}$ for 20 minutes at $4^{\circ} \mathrm{C}$. Plasma serum was collected and stored at $-80^{\circ} \mathrm{C}$. All blood samples were analyzed at the same time with ultra-pressure LC (UPLC)-MS. A $6 \mu$ l aliquot of each plasma sample was spiked with $6 \mu$ l mixture of deuteriumlabeled internal standards, which included nicotine-methyl- $\mathrm{D}_{3}$, cotinine-methyl- $\mathrm{D}_{3}$, and trans-3'-hydroxycotinine-methyl- $\mathrm{D}_{3}$, each at a concentration of $1 \mathrm{ppm}$. Fifty microliters of 100\% LC-MS-grade methanol was added to precipitate out proteins. After vortexing and subsequent centrifugation at $16,000 \mathrm{~g}$ for 15 minutes at $4^{\circ} \mathrm{C}, 35 \mu \mathrm{l}$ supernatant was transferred to a sample vial for analysis by UPLCMS analysis.

UPLC-MS/MS Conditions. Plasma samples prepared as described above were analyzed using an ABSciex 6500 LC-MS/MS system (Sciex, Framingham, MA), consisting of a LC-30AD UPLC pump, a SIL30 AC auto sampler, an ACQUITY UPLC BEH HILIC $\left(2.1 \mathrm{~mm} \times 100 \mathrm{~mm}, 1.7 \mu \mathrm{m}\right.$ particle size; Waters) column at $40^{\circ} \mathrm{C}$, and a tandem mass spectrometer (QTrap 6500). UPLC was performed at a flow rate of $0.4 \mathrm{ml} / \mathrm{min}$ using the following conditions: 1.5 minutes in $15 \%$ solvent $\mathrm{A}$, a linear gradient for 1 minute to $50 \%$ solvent A, 3 minutes in $50 \%$ solvent A, followed by a return to $15 \%$ solvent A for 2.5 minutes, where solvent $\mathrm{A}$ is $5 \mathrm{mM} \mathrm{NH}_{4} \mathrm{AC}(\mathrm{pH}$ 6.7) and
$50 \%$ acetonitrile $(\mathrm{v} / \mathrm{v})$, and solvent $\mathrm{B}$ is $5 \mathrm{mM} \mathrm{NH}_{4} \mathrm{AC}(\mathrm{pH}$ 6.7) and $90 \%$ acetonitrile $(\mathrm{v} / \mathrm{v})$. The injection volume of each plasma sample was $2 \mu \mathrm{l}$. The QTrap 6500 tandem mass spectrometer was equipped with an electrospray ionization probe operated in the positive-ion mode. The mass spectrometer was operated in the multiple reaction-monitoring mode, and the concentrations of all analytes were determined simultaneously under the following conditions: cotinine $m / z 177.1>98$, cotinine-methyl- $\mathrm{D}_{3} \mathrm{~m} / z 180.1>101$, trans-3'hydroxycotinine $(3 \mathrm{HC}) \mathrm{m} / z 193.1>80$, and 3HC-methyl-D $\mathrm{m} / z$ $196.1>80$. A collision energy of $27 \mathrm{~V}$ was used for cotinine and cotinine-methyl- $\mathrm{D}_{3}$, and $32 \mathrm{~V}$ for $3 \mathrm{HC}$ and $3 \mathrm{HC}$-methyl- $\mathrm{D}_{3}$. A dwell time of $100 \mathrm{~ms}$ was used for all analytes, as well as an ion-spray voltage of $5500 \mathrm{~V}$, an ion source gas flow of 50 , DP of $30 \mathrm{~V}, \mathrm{EP}$ of $10 \mathrm{~V}$, $\mathrm{CXP}$ of $10 \mathrm{~V}$, and source temperature of $450^{\circ} \mathrm{C}$. Standard curves were constructed by plotting the ratio of analyte peak area to peak area of the corresponding internal standard (described above) versus analyte concentration for eight analyte concentrations. Standards at concentrations ranging from 0.3 to $325.5 \mathrm{pg} / \mu \mathrm{l}$ were used to establish standard curves. Plasma analyte concentrations were determined by measuring the peak area ratios of analyte to internal standard and then calculating analyte concentration from the appropriate standard curve using MultiQuant 2.1.1 software. The quantification limits (signal/noise $>10$ ) for each compound were $0.3 \mathrm{pg} / \mu \mathrm{l}$ for cotinine and $0.6 \mathrm{pg} / \mu \mathrm{l}$ for $3 \mathrm{HC}$.

Open-Field Locomotor Test. The open-field chamber was composed of Plexiglas $(35 \mathrm{~cm} \mathrm{~L} \times 35 \mathrm{~cm} \mathrm{~W} \times 31 \mathrm{~cm} \mathrm{H}$ ), as described previously (Chen et al., 2018b). Following testing in the intravenous nicotine self-administration session, mice (Cohort $1, n=5$ ) were immediately transferred to the open-field chamber. After a 5-minute habituation period, subjects were scored in the open-field apparatus for a 15-minute session to assess locomotor activity. Activity was recorded with a video camera, and distance traveled, time mobile, and center time were scored by two experimenters blinded to the group condition with ANY-Maze Software (Stoelting, Wood Dale, IL).

Operant Food Training. Subjects ( $n=11$ males) were mildly food restricted to $85 \%$ to $90 \%$ of free feeding weight and trained to press a lever in an operant chamber (Med Associates) for food pellets (20 mg; TestDiet) up to a FR5TO20 sec schedule, as previously described (Fowler and Kenny, 2011). Each session was performed using two retractable levers (one active, one inactive). Completion of the response criteria on the active lever resulted in the delivery of a food pellet. Responses on the inactive lever were recorded but had no scheduled consequences. Once stable responding was achieved (criteria $>30$ pellets per session across three consecutive sessions), subjects were administered DLCI-1, or vehicle control, 30 minutes prior to the session utilizing a within-subject, crossover design. Subjects were provided at least one baseline day in between test sessions to ensure stable responding prior to the next injection in the series. Behavioral responses were automatically recorded by MedAssociates software.

Statistical Analyses. Data were analyzed by a $t$ test, one-way ANOVA, or two-way ANOVA, as appropriate, with Prism software (GraphPad, La Jolla, CA). Significant main or interaction effects were followed by Bonferroni post hoc comparison with correction for multiple comparisons. Statistical reporting for ANOVAs is presented as a F-statistic value: $F_{(\text {degrees of freedom for group/treatment number, degrees of }}$ freedom for subject number) $=$ test value. The criterion for significance was set at $\alpha=0.05$.

\section{Results}

Decreased Nicotine Self-Administration with Administration of DLCI-1. Mice were examined for the effects of DLCI-1 (Fig. 1A) or vehicle on intravenous nicotine selfadministration. Because nicotine is mainly metabolized by CYP2A6 in the liver, DLCI-1 or vehicle was administered via oral gavage to allow for first-pass absorption and thus more 

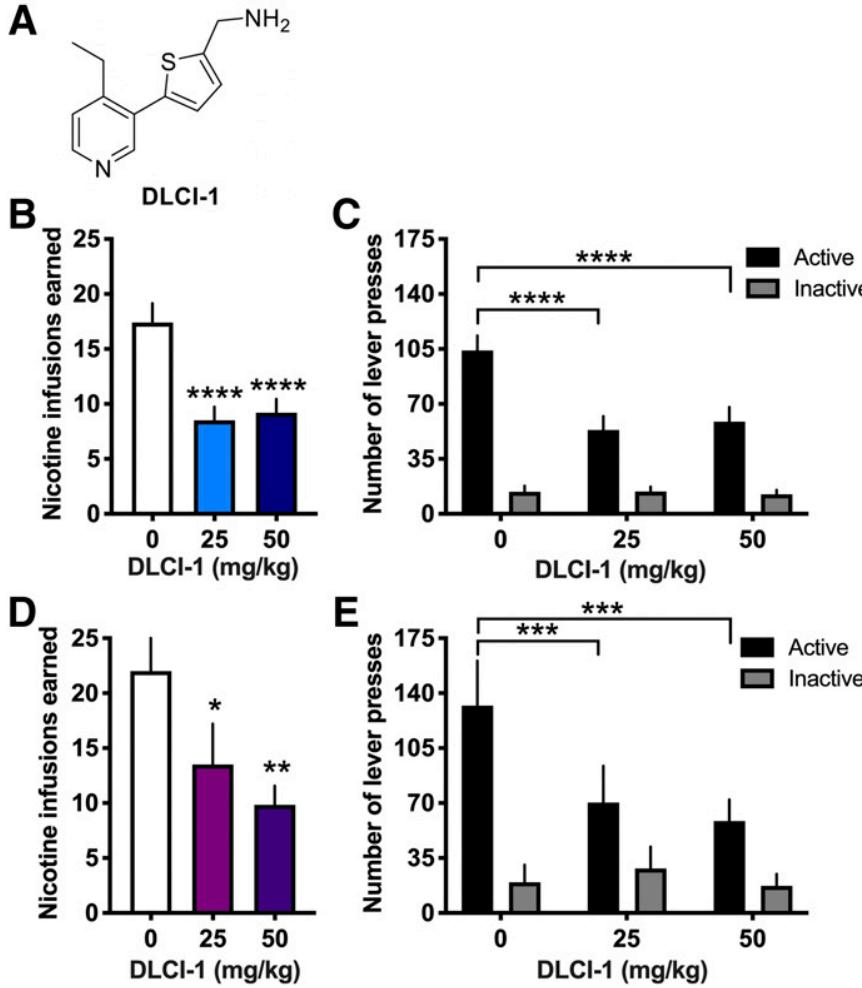

Fig. 1. The CYP2A6 inhibitor DLCI-1 decreases nicotine selfadministration in both male and female mice. Mice were examined for their responding to obtain intravenous nicotine infusions following administration of DLCI-1 or vehicle control. (A) Chemical structure of DLCI-1. (B) In males $(n=10)$, both doses of DLCI-1 ( 25 and $50 \mathrm{mg} / \mathrm{kg}$, by mouth) significantly decreased the number of nicotine infusions earned compared with vehicle control. (C) When the number of lever presses was examined for the nicotine sessions, male mice exhibited a significant decrease in the number of active lever presses with DLCI-1 pretreatment. In contrast, no significant differences were found in the number of inactive lever presses. (D) In females ( $n=6$ ), DLCI-1 decreased the number of nicotine infusions earned at both doses $(25$ and $50 \mathrm{mg} / \mathrm{kg}$, by mouth) compared with vehicle control. (E) Female mice also exhibited a significant decrease in the number of active lever presses with DLCI-1 pretreatment, but no decrease in the number of inactive lever presses. $* P<0.05 ; * * P<0.01$; *** $P<0.001$; **** $P<0.0001$ as compared with $0 \mathrm{mg} / \mathrm{kg}$ vehicle control. Data are presented as mean \pm S.E.M.

directed targeting to this organ. Administration of the 25 and $50 \mathrm{mg} / \mathrm{kg}$ doses of DLCI-1 effectively reduced the number of nicotine infusions earned compared with vehicle control in both males (Fig. 1B) (repeated-measures one-way ANOVA, $\left.\mathrm{F}_{(2,18)}=25.31, P<0.0001\right)$ and females (Fig. 1D) (repeatedmeasures one-way ANOVA, $\left.\mathrm{F}_{(2,10)}=9.88, P=0.0043\right)$. In the post hoc tests, both the 25 and $50 \mathrm{mg} / \mathrm{kg}$ doses resulted in significantly lower rates of responding than vehicle control for males $(P<0.0001)$ and females $(P<0.05$ vehicle vs. $25 \mathrm{mg} / \mathrm{kg}$, $P<0.01$ vehicle vs. $50 \mathrm{mg} / \mathrm{kg}$ ). To assess the level of leverpressing behavior, the number of active and inactive lever presses was also examined, and statistically significant differences were established for the active lever across conditions in both males (Fig. 1C) (repeated-measures two-way ANOVA, dose $\mathrm{F}_{(2,18)}=18.36, P<0.0001$; lever $\mathrm{F}_{(1,9)}=39.76, P=0.0001$; interaction $\mathrm{F}_{(2,18)}=22.33, P<0.0001$ ) and females (Fig. 1E) (repeated-measures two-way ANOVA, dose $\mathrm{F}_{(2,10)}=6.20$, $P=0.0177$; lever $\mathrm{F}_{(1,5)}=13.90, P=0.0136$; interaction $\mathrm{F}_{(2,10)}=$ $13.83, P=0.0013)$. Specifically, the vehicle injection resulted in a significantly greater number of active lever responses than both the 25 and $50 \mathrm{mg} / \mathrm{kg}$ doses for both males (post hoc, active 0 vs. $25, P<0.0001$; active 0 vs. $50, P<0.0001)$ and females (post hoc, active 0 vs. $25, P<0.001$; active 0 vs. $50, P<$ $0.001)$. Significant differences were not found in the rate of responding on the inactive levers. Together, these findings demonstrate that DLCI-1 effectively reduced nicotine intake, without affecting general nonspecific inactive lever-pressing behavior.

Blood samples were collected after nicotine selfadministration with DLCI-1 treatment to examine the levels of nicotine's main metabolite, cotinine, which is then metabolized into 3HC. If mice titrate their nicotine levels to maintain a similar level of activation of neural nAChRs across treatments, one would predict that the level of the metabolites would be relatively lower with inhibitor treatment across groups, despite differences in nicotine intake. This supposition considers the fact that each intravenous selfadministration session varies in the pattern of nicotine infusions across time and amount of nicotine administered, dependent on the animal's volitional behavior, which may also introduce the possibility of allowing for accumulation of nicotine with DLCI-1 treatment and thus similar levels of metabolites at the session end. Considerations must also be incorporated for differences in the rate of elimination of metabolites throughout the hour session duration via excretion from the body, with earlier infusions during the session likely resulting in greater excretion of metabolites prior to blood collection. Given this, we also analyzed the pattern of nicotine intake across treatment conditions to evaluate the time course of nicotine exposure for each treatment. In males (Fig. 2A), cotinine levels did not differ between the vehicle and $25 \mathrm{mg} / \mathrm{kg}$ treatment (paired $t$ test, $\mathrm{t}_{(6)}=1.506, P=0.0913$ ) or $50 \mathrm{mg} / \mathrm{kg}$ treatment (paired $t$ test, $\mathrm{t}_{(5)}=0.566, P=0.2979$ ). In contrast, $3 \mathrm{HC}$ levels were higher with vehicle compared with the $25 \mathrm{mg} / \mathrm{kg}$ treatment (paired $t$ test, $\mathrm{t}_{(6)}=2.816, P=0.0153$ ), but no differences were found between the vehicle and $50 \mathrm{mg} / \mathrm{kg}$ treatment (paired $t$ test, $\mathrm{t}_{(5)}=1.608, P=0.0844$ ). When examining nicotine intake across the 15 -minute intervals of the session, the vehicle condition maintained a consistently higher level of nicotine intake across the session, which was approximately twice that of the DLCI-1 treatments (Fig. 2B). In females (Fig. 2C), cotinine levels were statistically lower with both the $25 \mathrm{mg} / \mathrm{kg}$ treatment (paired $t$ test, $\mathrm{t}_{(5)}=2.465, P=0.0284$ ) and $50 \mathrm{mg} / \mathrm{kg}$ treatment (paired $t$ test, $\left.\mathrm{t}_{(5)}=3.101, P=0.0134\right)$, as compared with the vehicle. For $3 \mathrm{HC}$, differences were not found between the vehicle and $25 \mathrm{mg} / \mathrm{kg}$ treatment (paired $t$ test, $\mathrm{t}_{(5)}=1.768, P=0.0687$ ), but the $50 \mathrm{mg} / \mathrm{kg}$ treatment led to lower $3 \mathrm{HC}$ levels than the vehicle condition (paired $t$ test, $\mathrm{t}_{(5)}=4.670, P=0.0027$ ). When examining nicotine intake within the hour session in females, all conditions displayed a slight downward trajectory in nicotine consumption (Fig. 2D). Taken together, these findings are indicative of a dynamic process of intake, metabolism, and elimination for nicotine and its metabolites throughout the self-administration session.

Bupropion-Induced Decrease in Nicotine Self-Administration. To further support the potential of DLCI-1 as a tobacco cessation therapeutic, we assessed the effects of bupropion on nicotine intake for comparison purposes. Interestingly, the effects of bupropion on nicotine selfadministration in mice have not been previously investigated. Thus, we examined the impact of a moderate $(1 \mathrm{mg} / \mathrm{kg})$ and 

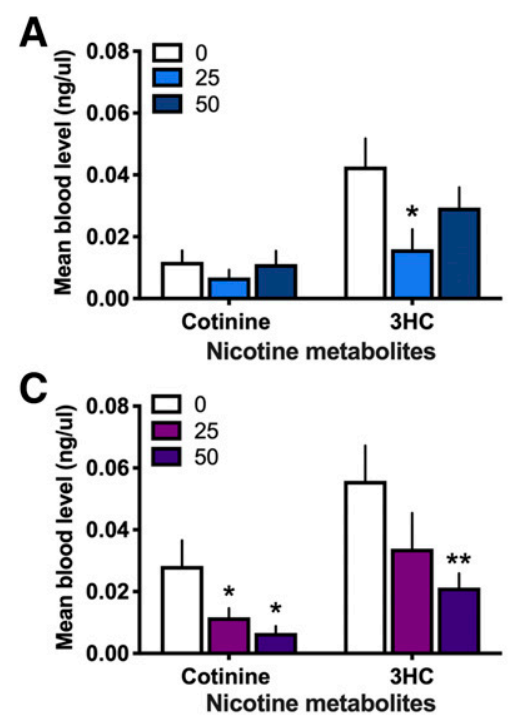
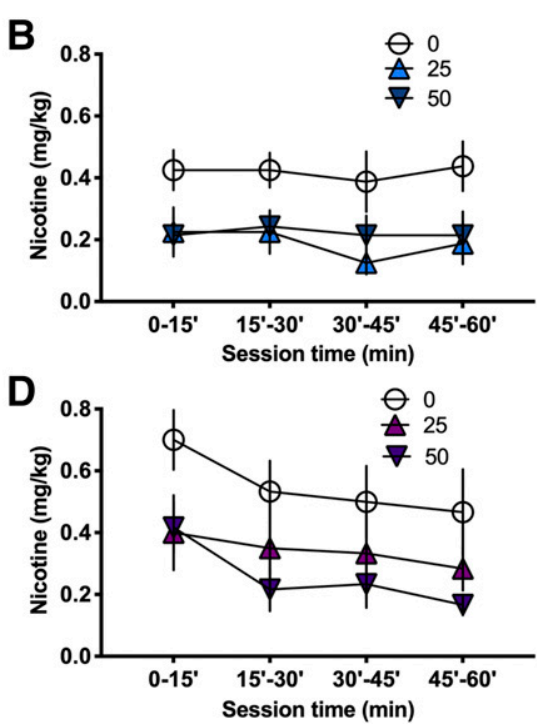

Fig. 2. Nicotine metabolites in the blood following DLCI-1 treatment and nicotine self-administration. Blood samples were analyzed following the 1-hour nicotine self-administration session to examine for differences in metabolite levels. (A) In males $(n=7)$, despite a higher level of nicotine intake in the vehicle condition, the levels of cotinine did not differ among treatments immediately after the session. However, the $25 \mathrm{mg} / \mathrm{kg}$ DLCI-1 treatment did lead to decreased 3HC levels compared with the vehicle treatment. (B) Analysis of the pattern of nicotine self-administered $(\mathrm{mg} / \mathrm{kg})$ throughout the session duration reveals that the males maintained a consistent level of responding over each 15-minute interval, with consistently lower intake found with DLCI1 treatment. (C) In females $(n=6)$, the 25 and $50 \mathrm{mg} / \mathrm{kg}$ DLCI-1 treatment resulted in a lower level of cotinine, and $50 \mathrm{mg} / \mathrm{kg}$ DLCI-1 also led to a significant decrease in 3HC, as compared with the vehicle treatment. (D) Analysis of the pattern of nicotine self-administered $(\mathrm{mg} / \mathrm{kg})$ throughout the session duration in females reveals that the vehicle and DLCI-1 treatments led to consistent levels of responding at higher and lower levels, respectively, and there was an apparent downward shift in intake over the 15-minute intervals. $* P<0.05$; $* * P<0.01$ compared with the $0 \mathrm{mg} / \mathrm{kg}$ vehicle control. high $(75 \mathrm{mg} / \mathrm{kg}$ ) bupropion dose; in the rodent literature, the doses of bupropion range from 0.1 to $78 \mathrm{mg} / \mathrm{kg}$ (Rauhut et al., 2003, 2008; Damaj et al., 2010; Grabus et al., 2012; Hall et al., 2015). The $1 \mathrm{mg} / \mathrm{kg}$ dose was selected based on prior findings that this dose abolishes nicotine reward in the conditioned place preference paradigm and reverses nicotine-induced tolerance in mice (Damaj et al., 2010; Grabus et al., 2012). The high $75 \mathrm{mg} / \mathrm{kg}$ dose was selected because this dose range has been shown to attenuate nicotine self-administration in rats in several prior studies (Rauhut et al., 2003, 2008; Hall et al., 2015). In males, we found a significant difference between treatments (Fig. 3A) (repeated-measures one-way ANOVA, $\left.\mathrm{F}_{(2,8)}=8.249, P=0.0114\right)$. Post hoc analyses revealed that the high dose $(75 \mathrm{mg} / \mathrm{kg})$, but not the moderate (1 $\mathrm{mg} / \mathrm{kg})$ dose, induced a significant decrease in nicotine intake compared with the vehicle $(P<0.01)$. However, it is important to note that during testing, the mice exhibited an adverse behavioral state following the high-dose bupropion injection, with behaviors representative of withdrawalshaking, hunched posture, gnawing, lethargy, and piloerection. As a quantitative measure, we examined the latency for the mice to receive their first nicotine infusion during the session, which provides evidence of the ability of the mice to ambulate around the testing chamber and press the lever. It is expected that subjects should attenuate their responding for nicotine with a therapeutic treatment after experiencing the interactive effects of the therapeutic with the first nicotine infusion; thus, subjects would not be expected to differ in the initial latency to first reward. However, we found a significant increase in the latency with the $75 \mathrm{mg} / \mathrm{kg}$ high-dose bupropion
A

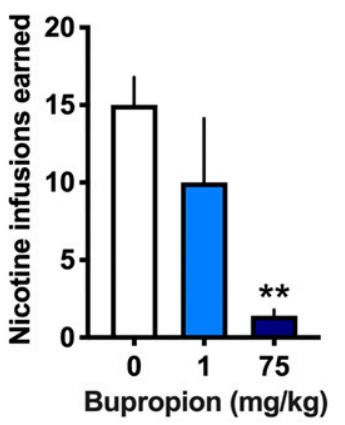

C

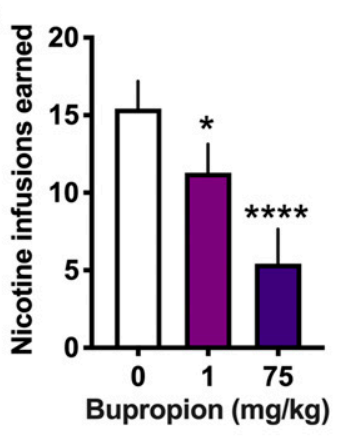

B

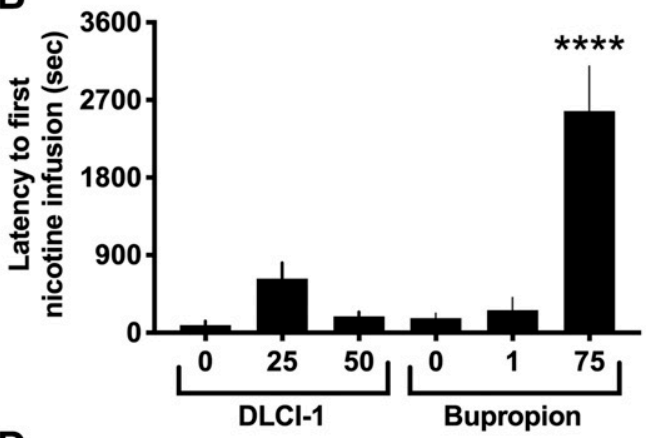

D

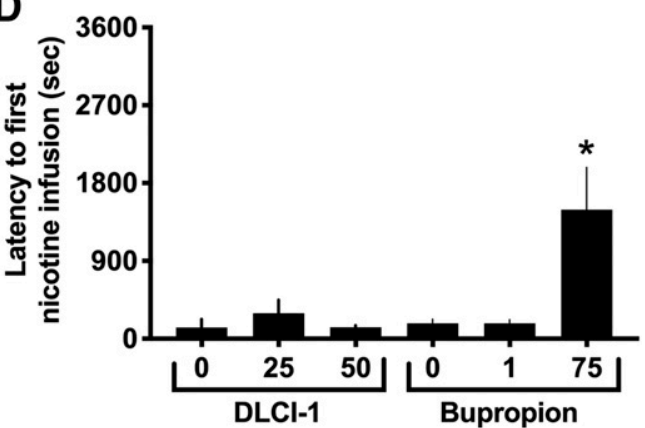

Fig. 3. Effects of bupropion treatment on nicotine selfadministration. Mice were examined to assess whether bupropion would exert a similar reduction in nicotine selfadministration as that found with DLCI-1. (A) In males $(n=5)$, a significant decrease in nicotine infusions earned was found following the high $(75 \mathrm{mg} / \mathrm{kg})$, but not moderate $(1 \mathrm{mg} / \mathrm{kg})$, bupropion treatment as compared with vehicle. $* * P<0.01$ vs. $0 \mathrm{mg} / \mathrm{kg}$ vehicle. (B) Males treated with the high dose of bupropion $(75 \mathrm{mg} / \mathrm{kg}$ ) exhibited significantly delayed latency to respond on the active lever for the first infusion of the session compared with all other treatment conditions, indicating behavioral suppression. $* * * * P<$ 0.0001 vs. all other treatment conditions. (C) In females $(n=7)$, both the moderate $(1 \mathrm{mg} / \mathrm{kg})$ and high $(75 \mathrm{mg} / \mathrm{kg})$ dose of bupropion significantly attenuated the number of nicotine infusions earned. ${ }^{* * * *} P<0.0001$ vs. $0 \mathrm{mg} / \mathrm{kg}$ vehicle. (D) Females administered the high dose of bupropion $(75 \mathrm{mg} / \mathrm{kg}$ ) had a significantly delayed latency to respond on the active lever for the first infusion of the session compared with all other treatments, indicating behavioral suppression. $* P<0.05$ vs. all other treatment conditions. 
treatment, but not for any other treatment condition with either DLCI-1 or bupropion, thereby providing evidence of the negative behavioral state of the male mice following the high bupropion dose (Fig. 3B) (one-way ANOVA, $\mathrm{F}_{(5,42)}=21.05, P<$ 0.0001 ; post hoc $P<0.0001$ for all conditions compared with $75 \mathrm{mg} / \mathrm{kg}$ bupropion). In females, bupropion at both doses resulted in a statistically significant decrease in nicotine intake relative to the vehicle (Fig. 3C) (repeated-measures one-way ANOVA, $\mathrm{F}_{(2,12)}=20.02, P=0.0002$; post hoc: vehicle vs. $1 \mathrm{mg} / \mathrm{kg}, P<0.05$; vehicle vs. $75 \mathrm{mg} / \mathrm{kg}, P<0.0001)$. At the high $75 \mathrm{mg} / \mathrm{kg}$ bupropion dose, similar adverse behavioral effects were noted in the females, which were further confirmed with analysis of the latency to first nicotine infusion among DLCI-1 and bupropion treatments (Fig. 3D) (one-way ANOVA, $\mathrm{F}_{(5,33)}=5.863, P=0.0006$; post hoc $P<0.05$ for all conditions compared with $75 \mathrm{mg} / \mathrm{kg}$ bupropion).

No Differences in Food Responding with Administration of DLCI-1. To further verify that the differences found with nicotine self-administration were selective to nicotine consumption, we examined the effects of DLCI-1 on operant responding for food pellets under the same FR5TO20 sec schedule. Separate cohorts of male mice were examined for the 25 and $50 \mathrm{mg} / \mathrm{kg}$ doses. At the $25 \mathrm{mg} / \mathrm{kg}$ dose, subjects did not differ in the amount of food pellets earned compared with vehicle control (Fig. $4 \mathrm{~A}$ ) (paired $t$ test, $\mathrm{t}_{(4)}=0.794, P=$ 0.4716 ), nor did they differ in the number of active or inactive lever presses between injection conditions (Fig. 4B) (repeatedmeasures two-way ANOVA, dose $\mathrm{F}_{(1,4)}=0.549, P=0.4996$; lever $\mathrm{F}_{(1,4)}=53.06, P=0.0019$; interaction $\mathrm{F}_{(1,4)}=0.662$, $P=0.4616$ ). Similarly, subjects did not differ at the $50 \mathrm{mg} / \mathrm{kg}$ dose in either food responding (Fig. 4C) (paired $t$ test, $\left.\mathrm{t}_{(5)}=1.416, P=0.2159\right)$ or number of active or inactive lever presses (Fig. 4D) (repeated-measures two-way ANOVA, dose $\mathrm{F}_{(1,5)}=2.525, P=0.1729$; lever $\mathrm{F}_{(1,5)}=339.6, P<0.0001$; interaction $\left.\mathrm{F}_{(1,5)}=2.093, P=0.2076\right)$. These data provide evidence that DLCI-1 does not affect lever-pressing behavior for food reward or general nonspecific inactive lever-pressing behavior.

Effects of DLCI-1-Induced Lowering of Nicotine Intake on Nicotine-Mediated Locomotion. Nicotine has been demonstrated to exert hyperlocomotive or hypolocomotive effects at low or high doses, respectively. Thus, following the nicotine session with the higher $50 \mathrm{mg} / \mathrm{kg}$ dose or vehicle control, mice were immediately transferred to open-field chambers to examine for differences in locomotion. This assessment serves as a secondary measure of DLCI-1's nicotine-mediated effects with the decreased selfadministered nicotine level. The mice consumed significantly less total nicotine (milligrams per kilogram) within each selfadministration session (Fig. 5A) (paired $t$ test, $\mathrm{t}_{(4)}=3.774, P=$ 0.0098). However, they did not differ in the total distance traveled within the open field (Fig. 5B) (paired $t$ test, $\mathrm{t}_{(4)}=$ $0.969, P=0.3874$ ) or in the total time mobile (Fig. 5C) (paired $t$ test, $\left.\mathrm{t}_{(4)}=0.433, P=0.6871\right)$. Furthermore, because decreased time in the center of an open field has been proposed to be a correlate of increased anxiety and nicotine has been shown to mediate anxiolytic behavioral effects, the duration of center time was assessed to provide an initial examination of an anxiety-associated response. However, the mice did not differ in the amount of time spent in the center of the open field (Fig. 5D) (paired $t$ test, $\mathrm{t}_{(4)}=0.185, P=0.8622$ ). These findings indicate that DLCI-1 decreases nicotine intake, and in doing so, results in similar nicotine-mediated behavioral actions with locomotion. Together, this provides evidence of inhibition of nicotine metabolism with similar in vivo actions of nicotine between conditions. Moreover, the food training and locomotor
A
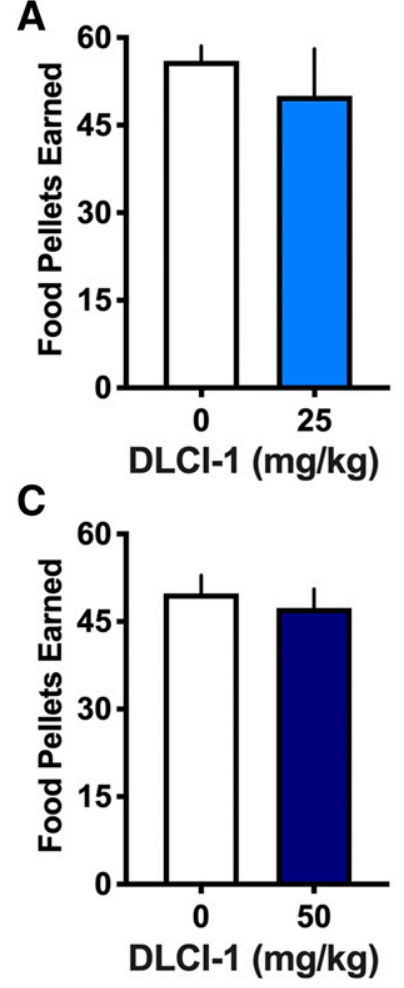

B

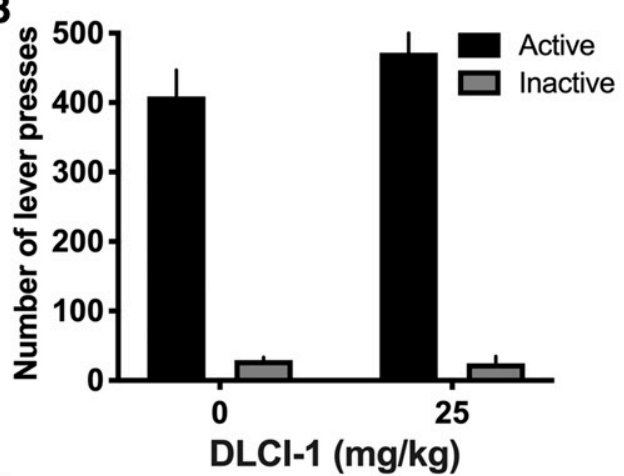

D

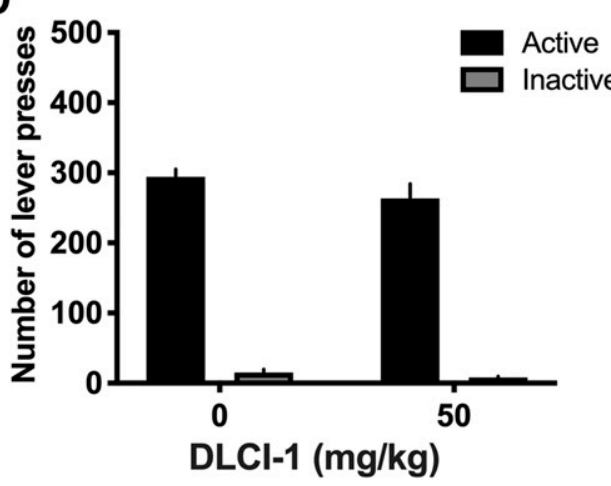

Fig. 4. No differences in food self-administration following administration of the CYP2A6 inhibitor DLCI-1. Mice were trained to self-administer food pellets and examined for the effects of DLCI-1 pretreatment on behavior ( $n=5$ to $6 /$ group). (A and B) When pretreated with the lower dose of DLCI-1 $(25 \mathrm{mg} / \mathrm{kg})$ or vehicle control, mice did not differ in the number of food pellets earned (A) or in the number of active and inactive lever presses (B). (C and D) Pretreatment with the higher dose of DLCI-1 (50 mg/kg) also did not induce any differences in food self-administration behavior for the number of pellets earned (C), or in the number of active and inactive lever presses (D). Data are presented as mean \pm S.E.M. 

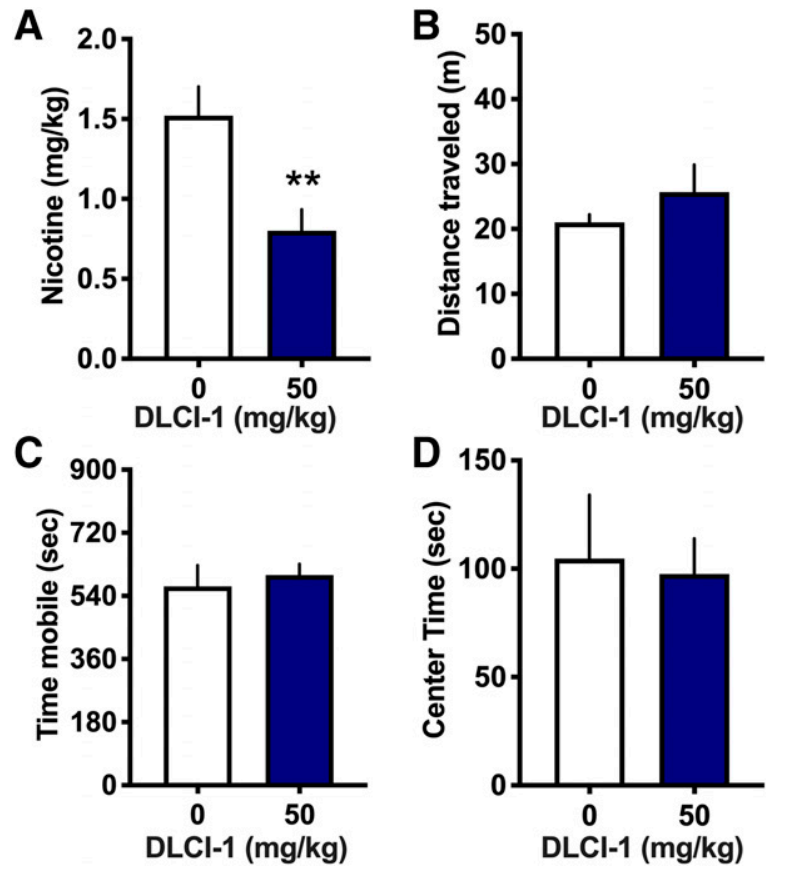

Fig. 5. Similar locomotor behaviors with DLCI-1 pretreatment despite decreased nicotine exposure. Following the intravenous nicotine selfadministration session with vehicle or $50 \mathrm{mg} / \mathrm{kg}$ DLCI-1 treatment, male mice $(n=5)$ were immediately examined for their locomotor behaviors in an open field. (A) During the nicotine session, DLCI-1 treatment resulted in approximately half of the amount of nicotine intake as that found in the control condition. $* * P<0.01$. (B-D) No significant differences were found in the total distance traveled (B), time mobile (C), or time spent in the center of the open field (D). Data are presented as mean \pm S.E.M.

data also support the contention that DLCI-1 does not induce any adverse behavioral effects at the doses administered.

\section{Discussion}

In these studies, we found that oral administration of DLCI1 , a potent, selective, and metabolically stable CYP2A6 inhibitor, results in decreased intravenous nicotine selfadministration in both male and female mice. Throughout the self-administration session, the mice appeared to consistently titrate their intake across the 15-minute session intervals. In males, this resulted in the detection of similar levels of nicotine's metabolites, cotinine and $3 \mathrm{HC}$, in the blood, with the exception of less $3 \mathrm{HC}$ found following the $25 \mathrm{mg} / \mathrm{kg}$ dose of DLCI-1. In contrast, females tended to adjust their responding downward throughout the session, and this resulted in significantly less cotinine and $3 \mathrm{HC}$ after the selfadministration session with the DLCI-1 treatments compared with vehicle. Interestingly, DLCI-1 induced an approximate $50 \%$ decrease on nicotine intake in both males and females, which was much greater than one of the first-line therapeutics for tobacco cessation, bupropion, at a moderate dose. Furthermore, the decrease in nicotine intake in the presence of DLCI1 led to a similar locomotor effect as that found with higher levels of nicotine intake under vehicle conditions, supporting the contention that similar levels of nAChR activation in the brain occurred despite decreased levels of nicotine intake with DLCI-1 treatment. Of further note, these effects were specific to lever pressing for nicotine reward, as differences in inactive lever pressing behavior or food self-administration were not found. Taken together, these data support the conclusion that the novel CYP2A6 enzyme inhibitor, DLCI-1, has therapeutic potential to selectively reduce tobacco and nicotine consumption and thereby promote smoking cessation.

The foundation for these studies is based on findings in humans with CYP2A6 allelic variation and prior reports with methoxsalen, in which lower levels of CYP2A6 nicotine metabolism have been associated with decreased nicotine intake and dependence (Sellers et al., 2000; Strasser et al., 2007; Patterson et al., 2008; Rodriguez et al., 2011; Liakoni et al., 2019). Methoxsalen is classified as a mechanism-based inhibitor; the drug itself becomes metabolically activated by CYP2A6, leading to an active metabolite that irreversibly binds to CYP2A6 and inhibits its activity (Blobaum, 2006). In addition, several studies in mouse models have provided further support for methoxsalen's potential in reducing nicotine dependence-associated behaviors (Alsharari et al., 2014; Bagdas et al., 2014). However, besides its actions on CYP2A6, methoxsalen also has carcinogen effects when combined with UV longwave radiation, and this has thereby limited its potential for further development as a smoking cessation therapeutic. In contrast, DLCI-1 was developed as a structural analog of nicotine; as such, DLCI-1 ascended from a leaddirected, medicinal chemical synthesis program with the highest CYP2A6 potency $(0.017 \mu \mathrm{M})$ of any inhibitor tested, to date, with high selectivity ratios $\left(\mathrm{IC}_{50 \text { (CYPXXX) }} / \mathrm{IC}_{50 \text { (CYP2A6) }}\right)$ toward all other human drug-metabolizing CYPs ranging from $>760$ for CYP3A4 to $>5$ for CYP2B6, and good human liver microsomal half-life (40 minutes). Furthermore, the compound falls within the boundaries of Lipinski's rule of five: calculated octanol-water partition coefficient $=2.108$, mol. wt. $=218.32$, topological polar surface area $=38.38$, number of hydrogen bond donors $=1$, and number of hydrogen bond acceptors $=2$ (Denton et al., 2018). Although the compound was designed to selectively inhibit CYP2A6, the possibility of DLCI- 1 to enter the brain by diffusing across the blood brain barrier may occur, which will need to be addressed in future studies.

It is of further note that the current studies were conducted in a mouse model system. Because rats metabolize nicotine primarily with CYP2B1, limitations are imposed when attempting to translate findings from rats to human hepatic function (Matta et al., 2007). In contrast, the mouse isoform CYP2A5 is homologous to human CYP2A6, allowing for a more relatable foundation for future clinical development. Furthermore, it should also be noted that DLCI-1 was dosed orally based on two considerations. First, given that nicotine is mainly metabolized in the periphery by the liver, this allowed us to take advantage of the first-pass effect found with gastrointestinal absorption, in which drug uptake occurs via the portal vein into hepatic circulation. Second, this route of administration provides translational relevance to future studies because oral administration is preferred for human dosing.

Interestingly, males and females demonstrated a similar effectiveness with the ability of DLCI- 1 to reduce nicotine selfadministration, as both sexes exhibited a decrease in the number of nicotine infusions and reduction of active lever presses with treatment. Thus, this effect was notably robust in consideration of the increased variability in responding typically found in female mice with intravenous nicotine self-administration. When assessing the amount of nicotine 
self-administered relative to nicotine's metabolites, differences with the DLCI-1 treatment were not consistent between sexes, which may be attributed to several factors. First, one must consider the fast metabolism of nicotine in mice relative to the session duration. Whereas humans exhibit a nicotine half-life of $\sim 2$ hours, the plasma nicotine metabolism of mice is estimated to be 6 to 7 minutes (Matta et al., 2007). In consideration of this time frame, the measurements in the current study reflect a dynamic process of varying intravenous nicotine infusions (dependent on the mouse's volitional behavior), metabolism, and excretion throughout the 1-hour session, given that blood samples were collected at the session end. Second, the rate of nicotine metabolism has been shown to differ between males and females. Specifically, females have been found to metabolize both nicotine and cotinine faster than males (Matta et al., 2007; Benowitz et al., 2009). In the current study, females exhibited more pronounced changes in nicotine metabolite levels with the treatmentinduced differences in nicotine self-administration, which is consistent with the prior findings. Third, chronic cigarette exposure has been shown to lead to decreased nicotine clearance in humans (Benowitz and Jacob, 1994; Benowitz et al., 2009), although it is unknown as to whether this can be attributed to nicotine alone (e.g., as opposed to other constituents in tobacco smoke) and whether mice develop similar changes with chronic exposure. Even so, given that the mice in the current study were chronically self-administering nicotine for at least 13 days prior to the DLCI-1 dosing schedule, our findings support the effectiveness of DLCI-1 as a potential therapeutic consistent with intervention after long-term nicotine exposure.

In an attempt to provide a correlate to the potential effectiveness of DLCI-I compared with other nicotine cessation therapeutics, we examined the effects of bupropion on intravenous nicotine self-administration in mice, which has not been previously investigated in the mouse model. Although the $1 \mathrm{mg} / \mathrm{kg}$ bupropion dose has been shown to block nicotine's rewarding effects in the conditioned place preference paradigm (Damaj et al., 2010), this dose was ineffective in altering nicotine self-administration in male mice, whereas the female mice exhibited a slight decrease in responding at this dose. Thus, comparing the $\sim 50 \%$ decrease in nicotine intake found with DLCI-1 to this dose of bupropion, the evidence suggests that DLCI-1 would be more efficacious. However, because studies in rats have used a much higher bupropion dose, we also examined the effects of a $75 \mathrm{mg} / \mathrm{kg}$ dose. Although both males and females exhibited a substantial decrease in the number of nicotine infusions earned, these effects were most likely secondary to general behavioral inhibition and malaise because the mice receiving the high dose exhibited adverse health-related effects (e.g., shaking, lethargy, gnawing, piloerection, hunched posture). This behavioral inhibition was further confirmed by examining the latency to respond for the first nicotine infusion, in which mice treated with $75 \mathrm{mg} / \mathrm{kg}$ bupropion exhibited a substantial delay in pressing the lever for the first infusion compared with all other doses for both DLCI-1 and bupropion. Moreover, the behavioral characteristics evidenced in the mice at this high dose are consistent with that found in humans with bupropion overdose (Stall et al., 2014). Interestingly, doses of bupropion in the intermediate range lead to increased, rather than decreased, nicotine self-administration in rats (Rauhut et al., 2003). Thus, taken together, these findings support the contention that DLCI-1 may serve as an effective therapeutic to promote tobacco and nicotine cessation.

In addition to the effectiveness on addiction-related processes, CYP2A6 inhibitors may also be relevant to tobaccoand nicotine-associated disease. For instance, polymorphism in the CYP2A6 gene rendering an absence of CYP2A6 metabolic function leads to decreased tobacco use and lung cancer risk (Ariyoshi et al., 2002). Conversely, an interaction between allelic variation in the CHRNA5-CHRNA3-CHRNB4 gene cluster, which encodes the $\alpha 5, \alpha 3$, and $\beta 4 \mathrm{nAChR}$ subunits, respectively, and normal CYP2A6 metabolism was found to increase risk for lung cancer, an effect that was interestingly found with both high and lower (e.g., $<20$ cigarettes per day) nicotine exposure (Wassenaar et al., 2011). Therefore, the resulting effects of CYP2A6 inhibition may serve to mitigate lung cancer risk in patients based on enzymatic levels and genetic predisposition.

In conclusion, the novel CYP2A6 inhibitor, DLCI-1, is a promising CYP2A6 inhibitor, which was rationally designed to decrease nicotine intake. Our data provide the first evidence that DLCI-1 effectively attenuates intravenous nicotine selfadministration in a mouse model. In addition to reducing nicotine consumption, pharmacological CYP2A6 inhibition may also lessen withdrawal symptoms to further promote abstinence and/or may ease the transition to achieve abstinence in combination with nicotine replacement approaches, although this needs to be further investigated. Taken together, these studies provide a foundation for further compound development to derive a highly efficacious therapeutic to promote tobacco, as well as e-cigarette, cessation.

\section{Authorship Contributions}

Participated in research design: Denton, C. D. Fowler.

Conducted experiments: Chen, J. P. Fowler, Watson, Sherafat, Staben, C. D. Fowler.

Contributed new reagents or analytic tools: Wang, Lazarus, Denton.

Performed data analysis: Chen, J. P. Fowler, Watson, Staben, Lazarus, C. D. Fowler.

Wrote or contributed to the writing of the manuscript: Chen, J. P. Fowler, Wang, Watson, Sherafat, Staben, Lazarus, Denton, C. D. Fowler.

\section{References}

Albuquerque EX, Pereira EF, Castro NG, Alkondon M, Reinhardt S, Schröder H, and Maelicke A (1995) Nicotinic receptor function in the mammalian central nervous system. Ann $N$ Y Acad Sci 757:48-72

Alsharari SD, Siu EC, Tyndale RF, and Damaj MI (2014) Pharmacokinetic and pharmacodynamics studies of nicotine after oral administration in mice: effects of methoxsalen, a CYP2A5/6 inhibitor. Nicotine Tob Res 16:18-25.

Ariyoshi N, Miyamoto M, Umetsu Y, Kunitoh H, Dosaka-Akita H, Sawamura Y, Yokota J, Nemoto N, Sato K, and Kamataki T (2002) Genetic polymorphism of CYP2A6 gene and tobacco-induced lung cancer risk in male smokers. Cancer Epidemiol Biomarkers Prev 11:890-894.

Audrain-McGovern J, Al Koudsi N, Rodriguez D, Wileyto EP, Shields PG, and Tyndale RF (2007) The role of CYP2A6 in the emergence of nicotine dependence in adolescents. Pediatrics 119:e264-e274.

Bagdas D, Muldoon PP, Zhu AZ, Tyndale RF, and Damaj MI (2014) Effects of methoxsalen, a CYP2A5/6 inhibitor, on nicotine dependence behaviors in mice. Neuropharmacology 85:67-72.

Benowitz NL, Hukkanen J, and Jacob P III (2009) Nicotine chemistry, metabolism, kinetics and biomarkers. Handb Exp Pharmacol 192:29-60.

Benowitz NL and Jacob P III (1994) Metabolism of nicotine to cotinine studied by a dual stable isotope method. Clin Pharmacol Ther 56:483-493.

Blobaum AL (2006) Mechanism-based inactivation and reversibility: is there a new trend in the inactivation of cytochrome p450 enzymes? Drug Metab Dispos 34:1-7. Bloom J, Hinrichs AL, Wang JC, von Weymarn LB, Kharasch ED, Bierut LJ, Goate A, and Murphy SE (2011) The contribution of common CYP2A6 alleles to variation in nicotine metabolism among European-Americans. Pharmacogenet Genomics 21: 403-416. 
Centers for Disease Control and Prevention (CDC) (2008) Smoking-attributable mortality, years of potential life lost, and productivity losses--United States, 20002004. MMWR Morb Mortal Wkly Rep 57:1226-1228.

Chen A, Krebs NM, Zhu J, and Muscat JE (2018a) Nicotine metabolite ratio predicts smoking topography: the Pennsylvania Adult Smoking Study. Drug Alcohol De pend 190:89-93.

Chen E, Lallai V, Sherafat Y, Grimes NP, Pushkin AN, Fowler JP, and Fowler CD (2018b) Altered baseline and nicotine-mediated behavioral and cholinergic profiles in ChAT-Cre mouse lines. J Neurosci 38:2177-2188.

Damaj MI, Grabus SD, Navarro HA, Vann RE, Warner JA, King LS, Wiley JL, Blough BE, Lukas RJ, and Carroll FI (2010) Effects of hydroxymetabolites of bupropion on nicotine dependence behavior in mice. $J$ Pharmacol Exp Ther 334: 1087-1095.

Denton TT, Srivastava P, Xia Z, Chen G, Watson CJW, Wynd A, and Lazarus P (2018) Identification of the 4-position of 3-alkynyl and 3-heteroaromatic substituted pyridine methanamines as a key modification site eliciting increased potency and enhanced selectivity for cytochrome P-450 2A6 inhibition. J Med Chem 61:7065-7086

Etter JF and Bullen C (2014) A longitudinal study of electronic cigarette users. Addict Behav 39:491-494.

Fowler CD and Kenny PJ (2011) Intravenous nicotine self-administration and cueinduced reinstatement in mice: effects of nicotine dose, rate of drug infusion and prior instrumental training. Neuropharmacology 61:687-698.

Fowler CD and Kenny PJ (2014) Nicotine aversion: neurobiological mechanisms and relevance to tobacco dependence vulnerability. Neuropharmacology 76:533-544

Fowler CD, Lu Q, Johnson PM, Marks MJ, and Kenny PJ (2011) Habenular $\alpha 5$ nicotinic receptor subunit signalling controls nicotine intake. Nature 471:597-601. GBD 2015 Tobacco Collaborators (2017) Smoking prevalence and attributable disease burden in 195 countries and territories, 1990-2015: a systematic analysis from the Global Burden of Disease Study 2015 [published correction appears in Lance (2017) 390:1644]. Lancet 389:1885-1906.

Gornall $J$ (2015) Why e-cigarettes are dividing the public health community. BMJ 350:h3317.

Grabus SD, Carroll FI, and Damaj MI (2012) Bupropion and its main metabolite reverse nicotine chronic tolerance in the mouse. Nicotine Tob Res 14:1356-1361.

Hall BJ, Slade S, Wells C, Rose JE, and Levin ED (2015) Bupropion-varenicline interactions and nicotine self-administration behavior in rats. Pharmacol Biochem Behav 130:84-89.

Léna C and Changeux JP (1998) Allosteric nicotinic receptors, human pathologies. $J$ Physiol Paris 92:63-74.

Liakoni E, Edwards KC, St Helen G, Nardone N, Dempsey DA, Tyndale RF, and Benowitz NL (2019) Effects of nicotine metabolic rate on withdrawal symptoms and response to cigarette smoking after abstinence. Clin Pharmacol Ther 105:641-651.

Matta SG, Balfour DJ, Benowitz NL, Boyd RT, Buccafusco JJ, Caggiula AR, Craig CR, Collins AC, Damaj MI, Donny EC, et al. (2007) Guidelines on nicotine dose selection for in vivo research. Psychopharmacology (Berl) 190:269-319.

Mwenifumbo JC, Lessov-Schlaggar CN, Zhou Q, Krasnow RE, Swan GE, Benowitz NL, and Tyndale RF (2008) Identification of novel CYP2A6*1B variants: the CYP2A6*1B allele is associated with faster in vivo nicotine metabolism. Clin Pharmacol Ther 83:115-121.

Patterson F, Schnoll RA, Wileyto EP, Pinto A, Epstein LH, Shields PG, Hawk LW, Tyndale RF, Benowitz N, and Lerman C (2008) Toward personalized therapy for smoking cessation: a randomized placebo-controlled trial of bupropion. Clin Pharmacol Ther 84:320-325.

Primack BA, Soneji S, Stoolmiller M, Fine MJ, and Sargent JD (2015) Progression to traditional cigarette smoking after electronic cigarette use among US adolescents and young adults. JAMA Pediatr 169:1018-1023.

Rauhut AS, Hawrylak M, and Mardekian SK (2008) Bupropion differentially alters the aversive, locomotor and rewarding properties of nicotine in CD-1 mice. Pharmacol Biochem Behav 90:598-607.

Rauhut AS, Neugebauer N, Dwoskin LP, and Bardo MT (2003) Effect of bupropion on nicotine self-administration in rats. Psychopharmacology (Berl) 169:1-9.

Raunio H and Rahnasto-Rilla M (2012) CYP2A6: genetics, structure, regulation, and function. Drug Metabol Drug Interact 27:73-88.

Ray R, Tyndale RF, and Lerman C (2009) Nicotine dependence pharmacogenetics: role of genetic variation in nicotine-metabolizing enzymes. J Neurogenet 23:252-261.

Rodriguez S, Cook DG, Gaunt TR, Nightingale CM, Whincup PH, and Day IN (2011) Combined analysis of CHRNA5, CHRNA3 and CYP2A6 in relation to adolescent smoking behaviour. J Psychopharmacol 25:915-923.

Sellers EM, Kaplan HL, and Tyndale RF (2000) Inhibition of cytochrome P450 2A6 increases nicotine's oral bioavailability and decreases smoking. Clin Pharmacol Ther 68:35-43.

Stall N, Godwin J, and Juurlink D (2014) Bupropion abuse and overdose. CMAJ 186: 1015

Strasser AA, Benowitz NL, Pinto AG, Tang KZ, Hecht SS, Carmella SG, Tyndale RF, and Lerman CE (2011) Nicotine metabolite ratio predicts smoking topography and carcinogen biomarker level. Cancer Epidemiol Biomarkers Prev 20:234-238.

Strasser AA, Malaiyandi V, Hoffmann E, Tyndale RF, and Lerman C (2007) An association of CYP2A6 genotype and smoking topography. Nicotine Tob Res 9:511-518.

Thorgeirsson TE, Gudbjartsson DF, Surakka I, Vink JM, Amin N, Geller F, Sulem P, Rafnar T, Esko T, Walter S, et al.; ENGAGE Consortium (2010) Sequence variants at CHRNB3-CHRNA6 and CYP2A6 affect smoking behavior. Nat Genet 42 448-453.

Tuesta LM, Fowler CD, and Kenny PJ (2011) Recent advances in understanding nicotinic receptor signaling mechanisms that regulate drug self-administration behavior. Biochem Pharmacol 82:984-995.

von Weymarn LB, Retzlaff C, and Murphy SE (2012) CYP2A6- and CYP2A13catalyzed metabolism of the nicotine $\Delta 5^{\prime}\left(1^{\prime}\right)$ iminium ion. $J$ Pharmacol Exp Ther 343:307-315.

Wassenaar CA, Dong Q, Wei Q, Amos CI, Spitz MR, and Tyndale RF (2011) Relationship between CYP2A6 and CHRNA5-CHRNA3-CHRNB4 variation and smoking behaviors and lung cancer risk. J Natl Cancer Inst 103:1342-1346.

Address correspondence to: Dr. Christie D. Fowler, Department of Neurobiology and Behavior, University of California Irvine, 1232 McGaugh Hall, Irvine, CA 92697-4550. E-mail: cdfowler@uci.edu; Dr. Travis T. Denton, Department of Pharmaceutical Sciences, College of Pharmacy and Pharmaceutical Sciences, Washington State University, 412 East Spokane Falls Boulevard, Spokane, WA 99202. E-mail: travis.denton@wsu.edu; or Dr. Philip Lazarus, Department of Pharmaceutical Sciences, College of Pharmacy and Pharmaceutical Sciences, Washington State University, 412 East Spokane Falls Boulevard, Spokane, WA 99202. E-mail: phil.lazarus@wsu.edu 